OPEN ACCESS

Edited by:

David Holcman,

École Normale Supérieure, France

Reviewed by:

Tao Zhang,

Nankai University, China John E. Lewis,

University of Ottawa, Canada

${ }^{*}$ Correspondence:

Wei Ren

renwei@snnu.edu.cn

Xingang Wang

wangxg@snnu.edu.cn

Received: 28 June 2016 Accepted: 26 September 2016

Published: 19 October 2016

Citation:

Chen M, Ren W and Wang X (2016)

Depotentiation from Potentiated

Synaptic Strength in a Tristable

System of Coupled Phosphatase and

Kinase.

Front. Comput. Neurosci. 10:104 doi: 10.3389/fncom.2016.00104

\section{Depotentiation from Potentiated Synaptic Strength in a Tristable System of Coupled Phosphatase and Kinase}

\author{
Mengjiao Chen ${ }^{1}$, Wei Ren ${ }^{2 *}$ and Xingang Wang ${ }^{1 *}$ \\ ${ }^{1}$ School of Physics and Information Technology, Shaanxi Normal University, Xi'an, China, ${ }^{2}$ Key Laboratory of Modern \\ Teaching Technology, Ministry of Education, Shaanxi Normal University, Xi'an, China
}

Long-term potentiation (LTP) of synaptic strength is strongly implicated in learning and memory. On the other hand, depotentiation, the reversal of synaptic strength from potentiated LTP state to the pre-LTP level, is required in extinction of the obsolete memory. A generic tristable system, which couples the phosphatase and kinase switches, exclusively explains how moderate and high elevation of intracellular calcium concentration triggers long-term depression (LTD) and LTP, respectively. The present study, introducing calcium influx and calcium release from internal store into the tristable system, further show that significant elevation of cytoplasmic calcium concentration switches activation of both kinase and phosphatase to their basal states, thereby depotentiate the synaptic strength. A phase-plane analysis of the combined model was employed to explain the previously reported depotentiation in experiments and predict a threshold-like effect with calcium concentration. The results not only reveal a mechanism of NMDAR- and mGluR-dependent depotentiation, but also predict further experiments about the role of internal calcium store in induction of depotentiation and extinction of established memories.

Keywords: long-term potentiation, depotentiation, tristability, kinase, phosphatase

\section{INTRODUCTION}

Over the past decades, ample evidences support that long-lasting modifications of synaptic strength, including long-term potentiation (LTP) and long-term depression (LTD), are strongly implicated in learning and memory. Meanwhile, depotentiation, which refers to reversal of synaptic strength from potentiated LTP state to its pre-LTP level, is required by extinction of the obsolete memory. In the amygdala, cued fear-conditioning induces widespread synaptic strengthening (McKernan and Shinnick-Gallagher, 1997; Rogan et al., 1997; Rumpel et al., 2005). There is growing evidence to support that fear memories are transiently susceptible to erasure due to the ability of the same experience to reverse this form of synaptic strengthening (Kim et al., 2007; Clem and Huganir, 2010; Díaz-Mataix et al., 2011). A similar role of depotentiation is also found in hippocampaldependent memory extinction (Zhang et al., 2011; Wang and Zhang, 2012). Novelty acquisition is not only able to induce LTP (Li et al., 2003; Gruart et al., 2006; Whitlock et al., 2006; Madroñal et al., 2007), but also enables induction of depotentiation from previously established LTP (Xu et al., 1998; Manahan-Vaughan and Braunewell, 1999; Abraham et al., 2002; Straube et al., 2003; Collingridge et al., 2010). These findings indicate that experience or stimulation protocol resulting 
in LTP can further induce depotentiation. However, mechanisms for depotentiation induced by further application of stimulations for LTP induction still remain unclear.

There have been a number of substantial theoretical investigations of the multi-stable mechanism of phosphatase and kinase switches which could account for LTP and LTD. Early simulation works proposed that the bistable switch formed by kinase, $\mathrm{Ca}^{2+} / \mathrm{CaM}$ protein kinase II (CaMKII), autophosphorylation underlies the stable transition between the basal and LTP states (Lisman, 1989). Recently, the authors further show that autodephosphorylation of protein phosphatase $2 \mathrm{~A}$ (PP2A), one of the few serine/threonine-specific phosphatases, also forms a bistable switch, which could account for the stable transition between the basal and LTD states. Even more importantly, coupling of the phosphatase and kinase switches produces a tristable system, which exclusively explains how moderate and high elevation of intracellular calcium concentration triggers LTD and LTP, respectively. A phase-plane analysis was employed to understand the model graphically, in which nullclines at different calcium concentrations were plotted to show how the system moves during LTD and LTP induction and how it is finally stabilized after the induction, respectively ( $\mathrm{Pi}$ and Lisman, 2008). This tristable system provides a generic theoretical framework for understanding dynamic mechanisms for LTP and LTD. Depotentiation induced by LTP induction protocol from the established LTP state to its basal state, which may be triggered by even higher elevation of intracellular concentration, also needs to be studied theoretically.

In a number of experiments, it has been shown that biochemical machinery for depotentiation is also formed by the molecular processes responsible for LTP, including calcium influxes, activation of specific enzymes, and glutamate receptor trafficking. Blockage of depotentiation when the induction stimulation was delivered in the presence of an antagonist of $\mathrm{N}$ methyl-D-aspartic acid (NMDA) receptors (NMDARs) suggests that activation of NMDA receptors is required (Christie et al., 1995; Abraham and Huggett, 1997). Activation of metabotropic glutamate receptors (mGluRs) has also been shown required for depotentiation (Bikbaev et al., 2008; Qi et al., 2013). Besides membrane permeability (activation of $\mathrm{Ca}^{2+}$ channels), the amount of $\mathrm{Ca}^{2+}$ influx depends on the calcium ion concentration difference in the intracellular vs. extracellular spaces, which causes diffusion of calcium ion from high to low concentrations. So, in experiment, lowering extracellular calcium ion is applied as one way to reduce influx and intracellular calcium ion elevation. Stimulation is no longer able to induce depotentiation when it is delivered in a condition in which extracellular $\mathrm{Ca}^{2+}$ concentration is lower than normal (Abraham and Huggett, 1997), suggesting elevation of intracellular $\mathrm{Ca}^{2+}$ concentration is also required. In addition, phosphatase inhibitor can block depotentiation, showing involvement of protein phosphatase pathways in depotentiation induction (Kang-Park et al., 2003). The above experimentations strongly suggest that, after the establishment of LTP, further increase of intracellular calcium concentration, which could be initiated by calcium influxes mediated by NMDARs and mGluRs, may regulate phosphatase and kinase pathways and then switch synaptic strength back to its pre-LTP level.

In the present study, calcium influxes through NMDARs and calcium release from internal calcium stores by activation of mGluRs are introduced to the tristable system to simulate the experimentally observed depotentiation by using LTP-induction protocol. An phase-plane analysis of the dynamics for transition from LTP to its basal level is also provided.

\section{MODEL AND METHODS}

Our model contains two primary ingredients as schematized in Figure 1. The first is a mechanism through which the plasticity of synaptic strength can be depicted. Here, we adopt the tristable biochemical network described by Pi and Lisman (2008), which describes coupling of the CaMKII switch and PP2A switch in the postsynaptic site of synapses. The system yields three different stable states, corresponding to the basal, the LTP, and the LTD levels of synaptic strength, respectively. Transient moderate elevation of calcium concentration can induce LTD, while high elevation induces LTP. In this model, the values of phosphorylated CaMKII and dephosphorylated PP2A govern $\alpha$ amino-3-hydroxy-5-methyl-4-isoxazole-propionic acid (AMPA) receptors (AMPARs) trafficking which determines the number of AMPAR inserted in the postsynaptic membrane and hence the synaptic efficacy. A basal state (with basal AMPARs level) is determined by low concentrations of both phosphorylated CaMKII and dephosphorylated PP2A, a LTP state (with high AMPARs level) by high phosphorylated CaMKII and low dephosphorylated PP2A, and a LTD state (with low AMPARs level) by low phosphorylated CaMKII and high dephosphorylated PP2A, respectively. Switching between these states is controlled by cytoplasmic calcium concentration in the post synaptic site. These states are self-sustaining; once the system moves into one of these states, it remains there, although calcium concentration returns to the basal level.

The second key part of our model is a description of the cytoplasmic calcium dynamics in the post synaptic site, including both calcium accumulation and calcium extrusion. Calcium accumulation in cytoplasm is mainly dependent on calcium influxes through NMDARs and calcium release from internal calcium store [for example, in endoplasmic reticulum (ER)].

\section{Calcium Dynamics}

Postsynaptic calcium dynamics in cytoplasm and in stores are expressed by Kusters et al. (2005):

$$
\begin{aligned}
\operatorname{Vol}_{C y t} \frac{d\left[\mathrm{Ca}^{2+}\right]_{C y t}}{d t}= & A_{E R}\left(J_{I P_{3} R}+J_{I K E R}-J_{S E R C A}\right) \\
& +\frac{10^{-6}}{Z_{C a} F} I_{\text {NMDAR }}-\frac{\left[\mathrm{Ca}^{2+}\right]_{C y t}-\left[\mathrm{Ca}^{2+}\right]_{\text {basal }}}{\tau_{C a}} \\
\operatorname{Vol}_{E R} \frac{d\left[\mathrm{Ca}^{2+}\right]_{E R}}{d t}= & A_{E R}\left(-J_{I P_{3} R}-J_{\text {IKER }}+J_{\text {SERCA }}\right)
\end{aligned}
$$




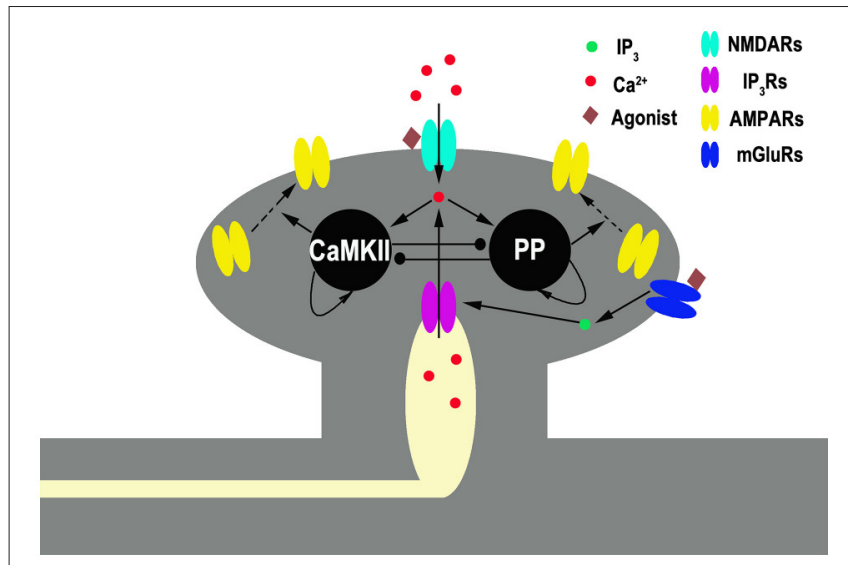

FIGURE 1 | Schematic diagram of the full model. Gray shading represents spine structure and light yellow shading represents internal stores [endoplasmic reticulum (ER)]. Pointed and circled arrows indicate the activation and the inhibition pathways, respectively. An agonist such as neurotransmitter binds to NMDARs and mGluRs. The latter one initiates a series of reactions, linked through G-protein, that ends in the production of the second messenger inositol trisphosphate $\left(I P_{3}\right)$, which diffuses through the cytoplasm and binds to $I P_{3}$ receptors $\left(I_{3} R s\right)$ locating at ER membrane. $I P_{3} R s$ are calcium channels binding with IP3 and mediating calcium release from the ER into cytoplasm. NMDARs are membrane calcium channels binding with glutamate mediating calcium influx. Cytoplasmic $\mathrm{Ca}^{2+}$ activates CaMKII (K) and protein phosphatase $(P)$ in a manner that depends on the concentration of cytoplasmic $\mathrm{Ca}^{2+}$. CaMKIl and phosphatase can self-activate themselves and inhibit each other. Active kinase and phosphatase control AMPARs insertion and removal, respectively.

Here, $I_{N M D A R}$ is the calcium current through NMDARs, $J_{I P_{3} R}$ denotes calcium flux from the ER to the cytoplasmic space through $\mathrm{IP}_{3} \mathrm{Rs}, J_{S E R C A}$ is the calcium flux pumped from the cytoplasmic space into the ER, $J_{I K E R}$ is the leak of calcium ions from the ER to cytoplasmic space, $Z_{C a}$ is calcium ion valence, $F$ is Faraday's constant, $\operatorname{Vol}_{C y t}$ is the volume of synapses, $\operatorname{Vol}_{E R}$ is the volume of stores, $A_{E R}$ is the surface area of the store exposed in synapses, $\left[\mathrm{Ca}^{2+}\right]_{E R}$ and $\left[\mathrm{Ca}^{2+}\right]_{C y t}$ are calcium concentrations of ER and cytoplasmic region, respectively, $\left[\mathrm{Ca}^{2+}\right]_{\text {basal }}$ is the basal concentration of calcium in cytoplasmic region and the decay constant is $\tau_{C a}$. The values of the different parameters in equations $(1,2)$ using in this paper are furnished in the Table 1.

$I_{N M D A R}$ is given by Shouval et al. (2002)

$$
\begin{aligned}
I_{N M D A R}= & P_{0} \cdot G_{N M D A R} \cdot \theta_{1}(t) \cdot\left\{I_{\text {glut }}^{f} \cdot \exp \left[\left(t_{\text {glut }}-t\right) / \tau_{\text {glut }}^{f}\right]\right. \\
& \left.+I_{\text {glut }}^{s} \cdot \exp \left[\left(t_{\text {glut }}-t\right) / \tau_{\text {glut }}^{s}\right]\right\} \cdot H(V)
\end{aligned}
$$

where $V$ is the postsynaptic potential and glutamate is released at a time $t=t_{\text {glut }}$. Here $P_{0}$ is the fraction of NMDARs that change to the open state after glutamate binding and $G_{N M D A R}$ is the conductance of the open NMDARs from calcium ions. $\theta_{1}$ is a step function: $\theta_{1}(t)=1$ when $t \geq t_{\text {glut }}$, and $\theta_{1}(t)=0$ when $t<t_{\text {glut }}$. Glutamate unbinding is characterized by two decay constants $\tau_{g l u t}^{f}$ and $\tau_{g l u}^{s}$, and the fast and slow unbinding components $I_{g l u t}^{f}$ and $I_{\text {glut }}^{s}$. The term $H(V)$ describes $\mathrm{Mg}^{2+}$ unblock due to changes

\begin{tabular}{|c|c|c|}
\hline Vol Cyt & $1 \times 10^{-12}$ & $\mathrm{dm}^{3}$ \\
\hline Vol $_{E R}$ & $0.1 \times 10^{-12}$ & $\mathrm{dm}^{3}$ \\
\hline$A_{E R}$ & $0.3 \times 10^{-7}$ & $\mathrm{dm}^{2}$ \\
\hline$Z_{C a}$ & 2 & - \\
\hline$F$ & 96,480 & $\mathrm{C} / \mathrm{mol}$ \\
\hline$\left[\mathrm{Ca}^{2+}\right]_{\text {basal }}$ & 0.1 & $\mu \mathrm{M}$ \\
\hline$\tau_{\mathrm{Ca}}$ & 12 & $\mathrm{~ms}$ \\
\hline
\end{tabular}

TABLE 1 | Parameter values of calcium dynamics in cytoplasmic region.

in the postsynaptic potential and is modeled by

$$
H(V)=\left(E_{C a}-V\right) /\left(1.0+\frac{\left[M g^{2+}\right] \cdot e^{-0.062 V}}{3.57}\right),
$$

where $E_{C a}$ is the reversal potential of calcium, $V$ is the postsynaptic membrane potential, and $\left[\mathrm{Mg}^{2+}\right]$ is the external magnesium ion concentration. All parameter values used for simulation of $I_{N M D A R}$ are listed in Table 2.

The expressions for each calcium fluxes from the store are

$$
\begin{array}{r}
J_{I P_{3} R}=f_{\infty}^{3} w^{3} K_{I P_{3} R}\left(\left[\mathrm{Ca}^{2+}\right]_{E R}-\left[\mathrm{Ca}^{2+}\right]_{C y t}\right), \\
J_{I K E R}=K_{I K E R}\left(\left[\mathrm{Ca}^{2+}\right]_{E R}-\left[\mathrm{Ca}^{2+}\right]_{C y t}\right), \\
J_{S E R C A}=J_{S E R C A}^{\max } \frac{\left[\mathrm{Ca}^{2+}\right]_{C y t}{ }^{2}}{K_{S E R C A}^{2}+\left[\mathrm{Ca}^{2+}\right]_{C y t}{ }^{2}},
\end{array}
$$

with

$$
f_{\infty}=\frac{\left[\mathrm{Ca}^{2+}\right]_{C y t}}{K_{f I P_{3}}+\left[C a^{2+}\right]_{C y t}} .
$$

And $w$ is the fraction of activated $\mathrm{IP}_{3} \mathrm{Rs}$; its dynamics is modeled by

$$
\frac{d w}{d t}=\frac{w_{\infty}-w}{\tau_{w}}
$$

with

$$
\begin{aligned}
w_{\infty} & =\frac{\frac{\left[I P_{3}\right]}{K_{w I P_{3}}+\left[I P_{3}\right]}}{\frac{\left[I P_{3}\right]}{K_{w I P_{3}}+\left[I P_{3}\right]}+K_{w(\mathrm{Ca})}\left[\mathrm{Ca}^{2+}\right]_{C y t}} \\
\tau_{w} & =\frac{a}{\frac{\left[I P_{3}\right]}{K_{w I P_{3}}+\left[I P_{3}\right]}+K_{w(\mathrm{Ca})}\left[\mathrm{Ca}^{2+}\right]_{C y t}} .
\end{aligned}
$$

$I P_{3}$ is used to mimic activation of mGluRs, we describe the change in $I P_{3}$ by production and degradation as follows (De Young and Keizer, 1992):

$$
\frac{d\left[I P_{3}\right]}{d t}=-I_{r}\left[I P_{3}\right]+I_{P} f(t)
$$

Where $\left[I P_{3}\right]$ is concentration of $I P_{3}$ in cytoplasm, $I_{r}$ is the rate constant for degradation of $I P_{3}, I_{p}$ is the maximal production generated by each activation of mGluRs, and $f(t)$ controls the timing of activation of mGluRs and is described by a step 
TABLE 2 | Parameter values of $I_{\text {NMDAR }}$.

\begin{tabular}{lcl}
\hline$P_{0}$ & 0.5 & - \\
$G_{N M D A}$ & 0.00035 & $\mu \mathrm{M} /(\mathrm{ms} \cdot \mathrm{mV})$ \\
$I_{\text {glut }}^{f}$ & 0.5 & - \\
$\tau_{\text {glut }}^{f}$ & 50 & $\mathrm{~ms}$ \\
$I_{\text {glut }}^{s}$ & 0.5 & - \\
$\tau_{\text {glut }}^{s}$ & 200 & $\mathrm{~ms}$ \\
$E_{\mathrm{Ca}}$ & 130 & $\mathrm{mV}$ \\
{$\left[\mathrm{Mg}^{2+}\right]$} & 1.5 & $\mathrm{mM}$ \\
\hline
\end{tabular}

function: If $t_{g l u t}+2 \mathrm{~ms}>t>t_{\text {glut }}, f(t)=1$; otherwise $f(t)=0$. Details of the parameters are listed in Table 3.

Postsynaptic potential change has been modeled by (Shouval et al., 2002)

$$
V=V_{\text {rest }}+E P S P
$$

where $V_{\text {rest }}$ is the resting membrane potential, $V_{\text {rest }}=-65 \mathrm{mV}$. EPSP is the net depolarized voltage generated by binding glutamate to receptors in post synapse. The EPSP is:

$$
E P S P=s \cdot\left[e^{\left(t_{\text {glut }}-t\right) / \tau_{1}}+e^{\left(t_{\text {glut }}-t\right) / \tau_{2}}\right],
$$

where $s$ is the maximal potential of depolarization, $s=50 \mathrm{mV}, \tau_{1}$ and $\tau_{2}$ are membrane potential decay constants, $\tau_{1}=50 \mathrm{~ms}$ and $\tau_{2}=5 \mathrm{~ms}$, and $t_{g l u t}$ is the time that glutamate released.

\section{$\mathrm{Pi}$ and Lisman Model of the CaMKII/PP2A Reaction Network}

In the present modeling, the CaMKII/PP2A reaction network exactly follows the work of $\mathrm{Pi}$ and Lisman (2008). The cytoplasmic calcium dynamics in the post synaptic site affects the rates of autocatalytic reactions that convert CaMKII and PP2A from their non-phosphorylated to phosphorylated forms. For CaMKII the concentration of the phosphorylated form, which is the active form, is denoted by $K^{*}$, while for PP2A the nonphosphorylated form is active, and its concentration is denoted by $P^{*}$. All equations describing the evolution of $K^{*}$ and $P^{*}$ were taken directly from Pi and Lisman (2008):

$$
\begin{aligned}
\frac{d}{d t} K^{*}= & k_{1} \frac{K_{t o t}-K^{*}}{K_{m 1}+\left(K_{t o t}-K^{*}\right)} K^{*}-k_{2} \frac{K^{*}}{K_{m 2}+K^{*}}\left(P^{*}+P_{0}\right) \\
& +k_{3} K_{0}+k_{4} \frac{\left[C a^{2+}\right]_{C y t}^{4}}{K_{m}^{4}+\left[C a^{2+}\right]_{C y t}^{4}}\left(K_{t o t}-K^{*}\right) \\
\frac{d}{d t} P^{*}= & k_{11} \frac{P_{t o t}-P^{*}}{K_{m 11}+\left(P_{t o t}-P^{*}\right)} P^{*}-k_{12} \frac{P^{*}}{K_{m 12}+P^{*}}\left(K^{*}+K_{0}\right) \\
& +k_{13} P_{0}+k_{14} \frac{\left[C a^{2+}\right]_{C y t}^{3}}{K_{m}^{3}+\left[C a^{2+}\right]_{C y t}^{3}}\left(P_{t o t}-P^{*}\right)
\end{aligned}
$$

where $k_{1}, k_{2}, k_{3}, k_{4}, k_{11}, k_{12}, k_{13}$, and $k_{14}$ are rate constants for each reaction. $K_{m 1}, K_{m 2}, K_{m}, K_{m 11}$, and $K_{m 12}$ are equilibrium constants for kinase and phosphatase. $K_{0}$ and $P_{0}$ indicate the basal concentration of active kinase and phosphatase. $K_{\text {tot }}$ and $P_{\text {tot }}$ indicate the total amount of kinase and phosphatase.
TABLE 3 | Parameter values of calcium dynamics in stores and IP dynamics.

\begin{tabular}{lcl}
\hline$K_{I P_{3} R}$ & $6 \times 10^{-5}$ & $\mathrm{dm} / \mathrm{s}$ \\
$K_{I K E R}$ & $0.002 \times 10^{-5}$ & $\mathrm{dm} / \mathrm{s}$ \\
$J_{S E R C A}^{\max }$ & $8 \times 10^{-5}$ & $\mu \mathrm{M} /\left(\mathrm{s} \cdot \mathrm{dm}^{2}\right)$ \\
$K_{S E R C A}$ & 0.2 & $\mu \mathrm{M}$ \\
$K_{f I P_{3}}$ & 0.5 & $\mu \mathrm{M}$ \\
$K_{W I P_{3}}$ & 1.5 & $\mu \mathrm{M}$ \\
$K_{W(C a)}$ & 0.5 & $\mu \mathrm{M}^{-1}$ \\
$I_{r}$ & 0.5 & $\mathrm{~s}^{-1}$ \\
$I_{p}$ & 0.009 & $\mu \mathrm{M}^{-1}$ \\
$a$ & 20 & $\mathrm{~s}$ \\
\hline
\end{tabular}

Dependence of AMPARs on $K^{*}$ and $P^{*}$ is described as:

$$
\frac{d}{d t} A=\left(c_{1} K^{*}+c_{3}\right)\left(A_{t o t}-A\right)-\left(c_{2} P^{*}+c_{4}\right) A,
$$

where $A$ and $A_{\text {tot }}$ indicate the AMPARs on the synaptic membrane and the total amount. $c_{1}$ and $c_{2}$ are scaling factors, $c_{3}$ and $c_{4}$ are rate constants for processes independent of kinase and phosphatase activities. All parameters of CaMKII/PP2A reaction network are listed in Table 4.

\section{Numerical Implementation}

All the calculations were implemented in the $\mathrm{C}$ programming language, with the differential equations expressed as different equations, and solved by using the fourth order Runge-Kutta method in steps of $0.01 \mathrm{~ms}$. According to the related experimental procedures, theta-burst stimulation (TBS) was used as triggers for elevation of calcium concentration in cytoplasm. One unit of TBS is consisted of 3 pulses $(100 \mathrm{~Hz})$ delivered at $200 \mathrm{~ms}$ intervals. For application of each specific units of TBS, the variation of cytoplasmic calcium concentration in the postsynaptic site, the values of concentration of active kinase and phosphatase, and synaptic efficacy were calculated as functions of time, respectively.

\section{RESULTS}

\section{Transitions of Synaptic Strength Induced by CaMKII/PP2A Network Switching}

To better understand the transition of synaptic strength, it is useful to consider how the original $\mathrm{Pi}$ and Lisman model switches in simpler situations. We begin with a study on the responses of the original CaMKII/PP2A network to a simple calcium pulse $\left(P_{C a}\right)$ with different amplitude and the same duration $(100 \mathrm{~ms})$. Simulation results are shown in Figure 2. The results for $P_{C a}$ $=4.5 \mu \mathrm{M}$ are consistent with those reported by Pi and Lisman (2008), changes of phosphatase and kinase activities are shown in Figure 2A. Both enzymes were nearly inactive in the basal state. During elevation of $P_{C a}$, the kinase and phosphatase become strongly activated. At the end of the induction period, $P_{C a}$ returns to the basal level, phosphatase activation returns back to its basal level subsequently but the kinase activation remains at a high level. In this way a LTP is induced. 
TABLE 4 | Parameter values of CaMKII/PP2A reaction network.

\begin{tabular}{lcl}
\hline$K_{\text {tot }}$ & 20 & $\mu \mathrm{M}$ \\
$P_{\text {tot }}$ & 20 & $\mu \mathrm{M}$ \\
$A_{\text {tot }}$ & 1 & - \\
$K_{0}$ & 0.5 & $\mu \mathrm{M}$ \\
$P_{0}$ & 0.5 & $\mu \mathrm{M}$ \\
$k_{1}$ & 2 & $\mathrm{~s}^{-1}$ \\
$k_{2}$ & 15 & $\mathrm{~s}^{-1}$ \\
$k_{3}$ & 1 & $\mathrm{~s}^{-1}$ \\
$k_{4}$ & 120 & $\mathrm{~s}^{-1}$ \\
$k_{11}$ & 2 & $\mathrm{~s}^{-1}$ \\
$k_{12}$ & 15 & $\mathrm{~s}^{-1}$ \\
$k_{13}$ & 1 & $\mathrm{~s}^{-1}$ \\
$k_{14}$ & 80 & $\mathrm{~s}^{-1}$ \\
$K_{m}$ & 4 & $\mu \mathrm{M}$ \\
$K_{m 1}$ & 10 & $\mu \mathrm{M}$ \\
$K_{m 2}$ & 0.3 & $\mu \mathrm{M}$ \\
$K_{m 11}$ & 10 & $\mu \mathrm{M}$ \\
$K_{m 12}$ & 1 & $\mu \mathrm{M}$ \\
$c_{1}$ & 1 & - \\
$c_{2}$ & 1 & - \\
$c_{3}$ & 6 & $\mathrm{~s}^{-1}$ \\
$c_{4}$ & 8 & $\mathrm{~s}^{-1}$ \\
\hline
\end{tabular}

However, further transient elevations of calcium concentration failed to induce a LTP state. The simulation result of changes in phosphatase and kinase activities to a transient increase of $P_{C a}$ to $6.5 \mu \mathrm{M}$ is shown in Figure 2B. Similarly, two enzymes are inactive in the basal state. During $P_{C a}$ elevation, the kinase and phosphatase also become strongly activated. At the end of the induction period, $P_{C a}$ returns to its basal level and both phosphatase and kinase return to their basal states subsequently. So there is no sustained change in synaptic strength.

To further characterize the transitions between different states, we systematically varied amplitude of $P_{C a}$ with the duration fixed at $100 \mathrm{~ms}$. For $P_{C a}$ between 0.1 and $1.3 \mu \mathrm{M}$, the system is stabled at basal state. For $P_{C a}$ in a range between 1.4 and $2.4 \mu \mathrm{M}$, the system is switched from the basal state to LTD states. For $P_{C a}$ between $3.0 \mu \mathrm{M}$ and $6.2 \mu \mathrm{M}$, it is switched to LTP states. However, in conditions with $P_{C a}>6.2 \mu \mathrm{M}$, neither LTP nor LTD is inducible (Figure 2C), the system returns to its basal state, suggesting that significant elevation of calcium concentration may depotentiate synaptic strength to basal levels.

Depotentiation from a LTP state was also tested by inputting a large $P_{C a}$ at a LTP level. As shown in Figure 2D, after a LTP is induced from the basal state by a proper $P_{C a}(4.5 \mu \mathrm{M}$ for $100 \mathrm{~ms})$, a large $P_{C a}(6.5 \mu \mathrm{M}$ for $100 \mathrm{~ms})$ reverses the potentiated state to its basal level, this mechanism of depotentiation is different from the ones induced by small elevations of $P_{C a}$ (Pi and Lisman, 2008), which induce LTD from a basal synaptic strength.

\section{The Amount of TBS Trains Determines Changes in Cytoplasmic Calcium Concentration, $K^{*}$, and $P^{*}$}

In order to directly simulate the experimental induction of depotentiation from a LTP state, we applied the induction procedure used in the experiments to the modified tristable system (including calcium dynamics) in simulation. A postsynaptic cytoplasmic calcium concentration dynamics produced by NMDARs activation and calcium release from internal calcium stores was considered and coupled in the original system. We then observed very different responses induced by application of different amount of TBS.

We first consider the responses to a brief TBS. A train of corresponding elevations of cytoplasmic calcium concentration is induced, and the amplitudes of these elevations are moderate (Figure 3A1). Details of the result are presented in Figure 3A2. Before application of the stimulation, no transmitters are released, thus NMDARs are closed, while the basal concentration of $I P_{3}$ is very low. The $\mathrm{IP}_{3} \mathrm{Rs}$ are also closed, therefore, there are neither $\mathrm{Ca}^{2+}$ influx through NMDARs nor $\mathrm{Ca}^{2+}$ release through $\mathrm{IP}_{3}$ Rs. During TBS delivery, NMDARs are activated to mediate a series of $\mathrm{Ca}^{2+}$ influx through NMDARs (Figure 3A2 top), while $\mathrm{IP}_{3} \mathrm{Rs}$ is still closed (Figure $\mathbf{3 A 2}$ bottom) as the concentration of $I P_{3}$ is not high enough, although it shows growing pulsatile elevations (Figure 3A2 middle). The results show that, a comparatively high elevation in cytoplasmic calcium concentration is induced by brief TBS, which is mainly mediated by activation of NMDARs.

As shown in Figure 3A3, before elevation of cytoplasmic calcium concentration, both enzymes are nearly inactive in the basal state. During the elevation of cytoplasmic calcium concentration, kinase and phosphates become strongly activated. Then cytoplasmic calcium concentration decline to the basal level, phosphates returns back to the basal level subsequently, while the kinase still stays at the activated state. Therefore, synaptic efficacy is switched to a LTP state.

Then, we turn to consider the responses to a prolonged TBS. Correspondingly, a series of elevations of cytoplasmic calcium concentration is induced by this prolonged TBS, and the amplitudes of the elevations gradually increase to significantly high levels (Figure 3B1). We then analyzed in details the sources of $\mathrm{Ca}^{2+}$. Before the stimulation, both NMDARs and $\mathrm{IP}_{3} \mathrm{Rs}$ are closed and there is no $\mathrm{Ca}^{2+}$ influx. As this prolonged TBS is delivered, NMDARs are activated to mediate stronger $\mathrm{Ca}^{2+}$ influx (Figure 3B2 top), meanwhile, $\mathrm{IP}_{3} \mathrm{Rs}$ are gradually activated (Figure 3B2 bottom) as the concentration of $I P_{3}$ is accumulated to a higher lever (Figure 3B2 middle). Therefore, a more significant elevation in cytoplasmic calcium concentration is eventually formed by activation of both NMDARs and $\mathrm{IP}_{3} \mathrm{Rs}$.

Changes in responses of phosphates and kinase caused by this significant elevation in cytoplasmic calcium concentration are shown in Figure 3B3. Both enzymes are nearly inactive in the basal state. During this significant elevation in cytoplasmic calcium concentration, the kinase and phosphates become strongly activated. However, both of them return back to their basal states subsequently as cytoplasmic calcium concentration declines to basal levels. Therefore, synaptic efficacy remains at the basal state.

\section{Reversal of an Established LTP}

Previous behavioral experiments have also shown that brief novelty acquisition was insufficient to reverse LTP, but a prolonged novelty exposure did reverse an established LTP 

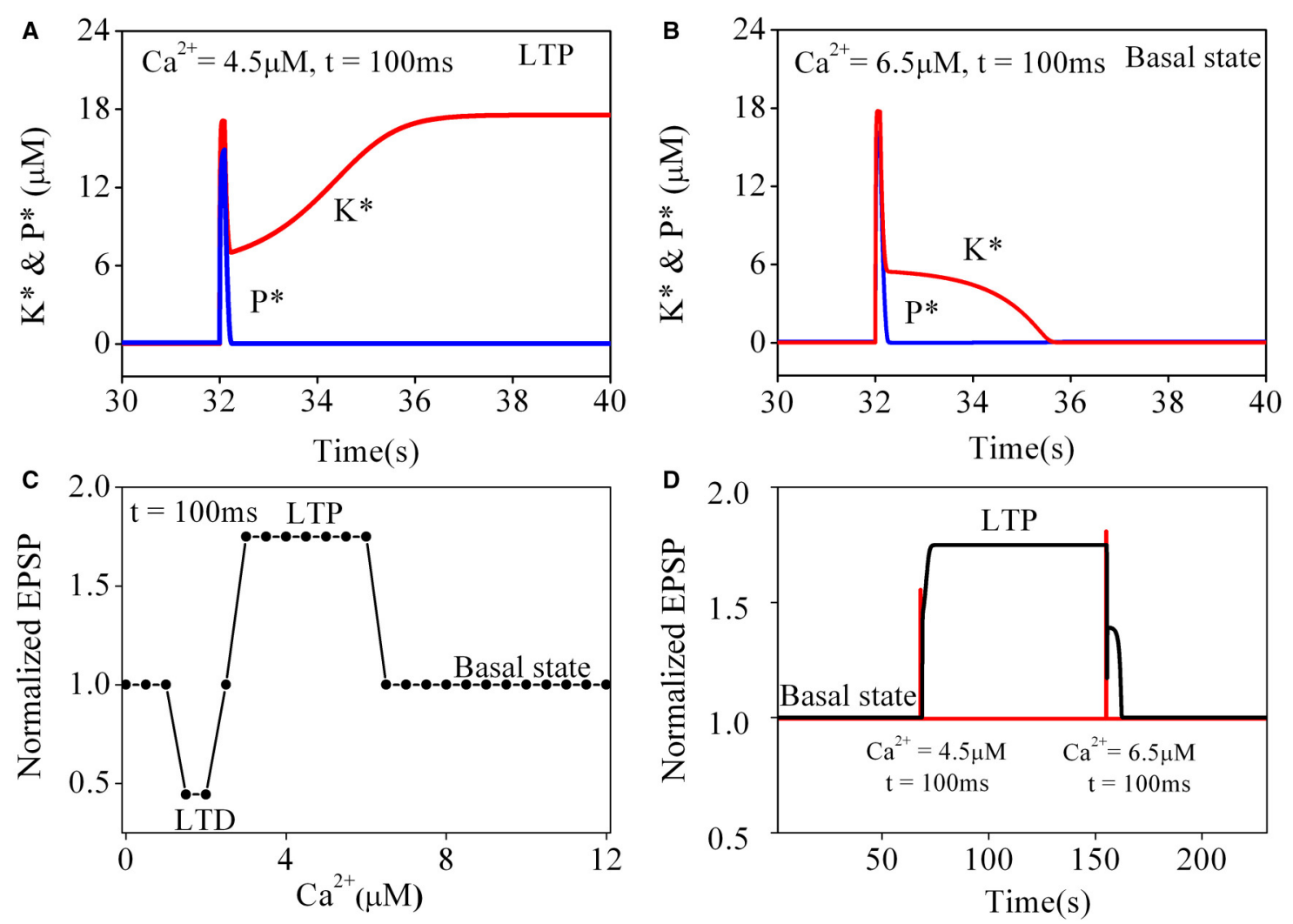

FIGURE 2 | Simulation results for original pi and lisman model of coupled kinase and phosphatase swithes in response to different simple calcium pulse. $K^{*}$ and $P^{*}$ denote concentration of phosphorylated kinase and dephosphorylated phosphatase, respectively. (A) Changes of $K^{*}$ and $P^{*}$ in response to application of $4.5 \mu \mathrm{M}$ calcium pulse $\left(P_{C a}\right)$ for $100 \mathrm{~ms}$. Before $P_{C a}$ stimulation, $K^{*}$ and $P^{*}$ both stay at basal level. During $P_{C a}$ application, $K^{*}$ and $P^{*}$ rise, and $K^{*}$ is dominate. After $P_{C a}$ is over, $P^{*}$ returns back to basal level while $K^{*}$ stays at high level, which finally leads to LTP. (B) Changes of $K^{*}$ and $P^{*}$ in response to application of $6.5 \mu \mathrm{M}$ calcium pulse for $100 \mathrm{~ms}$. Both $K^{*}$ and $P^{*}$ are transiently increased by $P_{\mathrm{Ca}}$ and return back to basal level after $\mathrm{Ca}^{2+}$ is removed. Therefore, synaptic efficacy remains unchanged. Red line represents $K^{*}$ and blue line for $P^{*}$, respectively. (C) Dependence of synaptic efficacy on amplitudes of $P_{C a}$. As the level of calcium increase from moderate to high, synaptic efficacy changes from LTD to LTP, then return to basal state when amplitude of $P_{C a}$ increases further. (D) Reversals of LTP. LTP is induced by the first stimulation $(4.5 \mu \mathrm{M}, 100 \mathrm{~ms})$ and reversed to basal level (depotentiation) by the second stimulation $(6.5 \mu \mathrm{M}, 100 \mathrm{~ms})$. Changes in $P_{\mathrm{Ca}}$ is shown with red line and synaptic efficacy with black line, respectively.

(Qi et al., 2013). Similar phenomena were also observed in hippocampus slices by using electrical stimulations. In the CA1 region of hippocampus slices from young adult rats, TBS, which mimics a physiologically relevant frequency of neuronal activity exhibited in the hippocampus of behaving animals (Kandel and Spencer, 1961; Ranck, 1973; Bikbaev et al., 2008), induces longlasting reversal of previously induced LTP (Barr et al., 1995). To test for whether a prolonged TBS can reverse LTP while a brief TBS cannot, we first simulated the effect of a brief TBS on established LTP. As shown in Figure 4A, activation of kinase was initially set at an activated state and phosphates at a basal state, corresponding to a LTP state of synaptic strength. When an elevation of cytoplasmic calcium concentration is induced by a brief TBS, kinase becomes slightly inactivated and phosphatase strongly activated. After cytoplasmic calcium concentration declines to the basal state, kinase returns to its initial activated state and phosphatase returns to its basal state. In other words, before and after brief TBS, activation levels of both kinase and phosphatase are unchanged. Hence, a brief TBS is not able to induce a transition from the established LTP state to the basal state.

Then, we simulated the effect of a prolonged TBS on an established LTP. The results are presented in Figure 4B. Before application of TBS, activation of kinase was still set at an activated state and phosphatase at a basal state. During a significant elevation by a prolonged TBS, kinase becomes strongly inactivated but phosphatase becomes strongly activated. When cytoplasmic calcium concentration declines to its basal level, activated forms of both kinase and phosphatase are switched to their basal states. The results clearly show that a prolonged TBS can reverse an established LTP by switching kinase from its activated state to its basal state, which is induced by a significant elevation of calcium concentration at the post synaptic site.

\section{Phase Space Analysis}

In order to show the dynamics of depotentiation graphically, the nullclines of CaMKII/PP2A network at different $\mathrm{Ca}^{2+}$ 
A

(1)

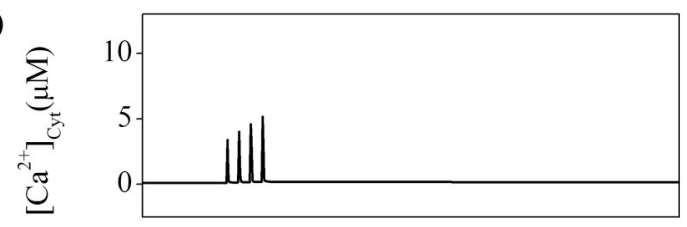

(2)
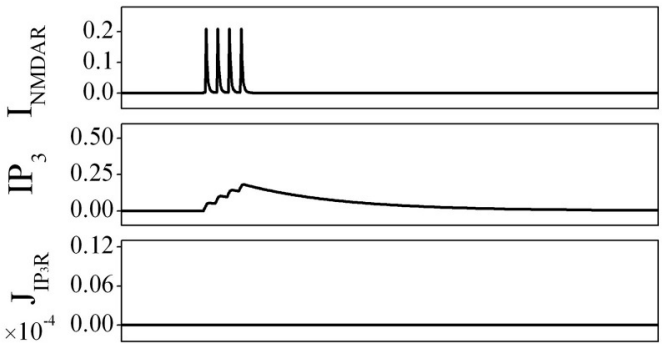

(3)

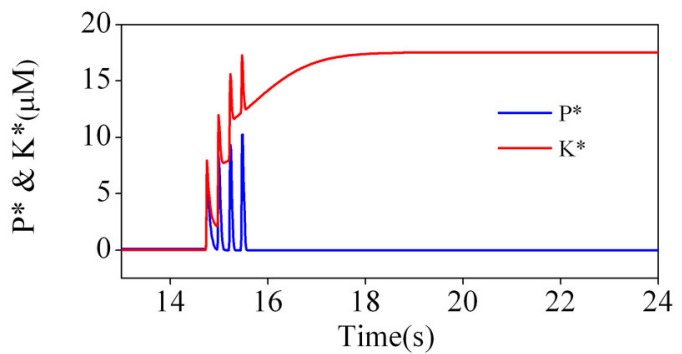

B

(1)

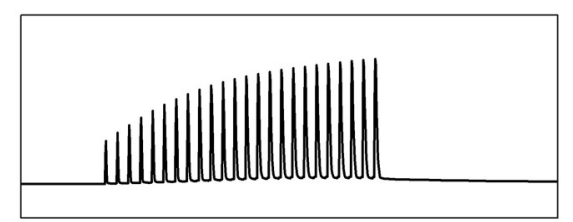

(2)
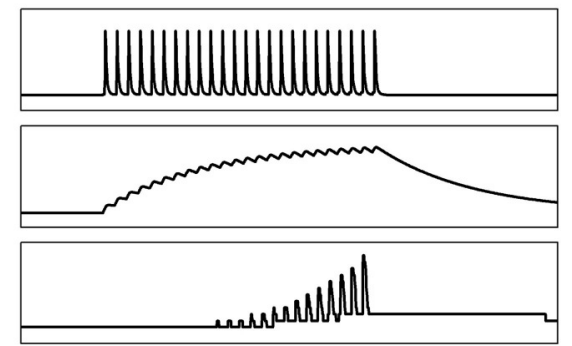

(3)

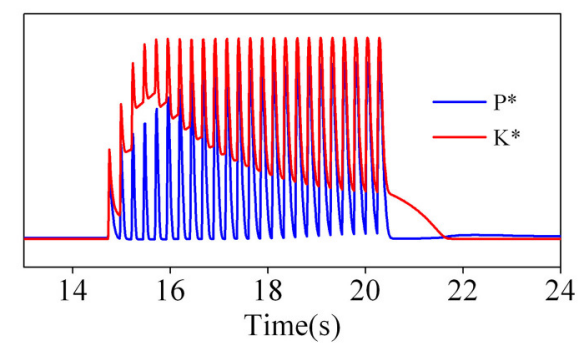

FIGURE 3 | The amount of TBS trains determines the changes in cytoplasmic calcium concentration, $\boldsymbol{K}^{\boldsymbol{*}}$, and $\boldsymbol{P}^{\boldsymbol{*}}$. Cytoplasmic calcium concentration in post-synapse induced by a brief TBS (4 trains) (A1) and a prolonged TBS (24 trains) (B1). Details of each calcium fluxes induced by the brief (A2) and the prolonged TBS (B2). Enzyme activation induced by the brief TBS (4 trains) (A3) and the prolonged TBS (B3). Before the brief TBS, both $K^{\star}$ and $P^{\star}$ are nearly inactive. During stimulation, both $K^{\star}$ and $P^{*}$ are transiently increased. After calcium removal, $K^{\star}$ stays at high and $P^{*}$ returns back to basal level. Therefore, LTP is induced. On the other hand, the prolonged TBS induces a depotentiation.
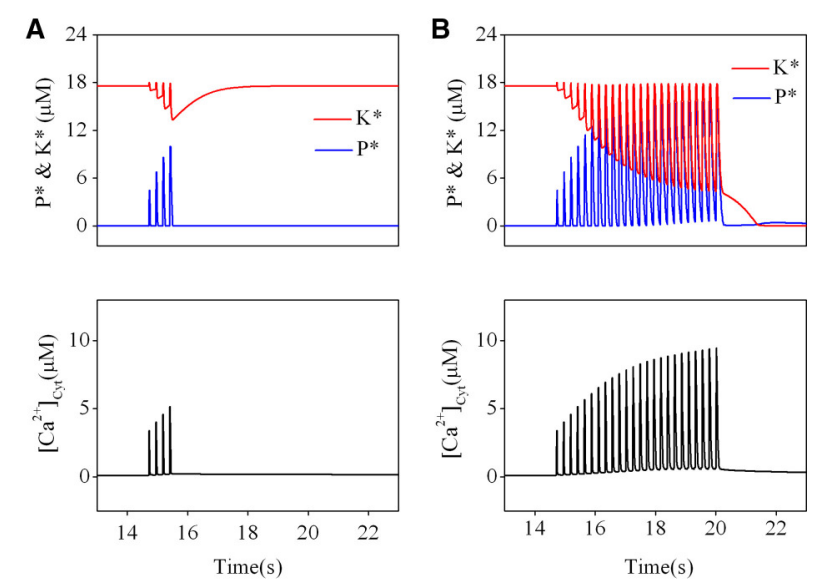

FIGURE 4 | Effect of different TBS on an established LTP. Changes of kinase $\left(K^{*}\right)$ and phosphatase $\left(P^{*}\right)$ (upper) and calcium dynamics (bottom) induced by the brief TBS (A) and the prolonged TBS (B).

concentrations is given in Figure 5. Firstly, we studied the case with unchanged synaptic efficacy after action of $\mathrm{Ca}^{2+}$ pulses. In this case, the system stays at the basal state before application of $\mathrm{Ca}^{2+}$ pulses. At the basal $\mathrm{Ca}^{2+}$ level, nullclines (Figure 5A, left panel) intersects at five points; three are stable points (filled circles at top left, bottom left, and bottom right) and two are unstable (open circles). In the basal state, the system is stable at the bottom left point. During a high $\mathrm{Ca}^{2+}$ elevation, the nullclines are deformed to create only one stable state (Figure 5A, middle panel, light blue and light red lines, and gray dot), and the system finally stays at the new unique stable state (Figure 5A, middle panel, gray trace). After action of $\mathrm{Ca}^{2+}$ pulses, the three stable states restore, but the system stays at the top left stable point (LTP) (Figure 5A, top right panel). However, during a significant elevation of $\mathrm{Ca}^{2+}$ concentration, nullclines are deformed to create another unique stable state (Figure 5A, middle panel, blue and red lines, and black dot), and the system moves from this stable state (Figure 5A, middle panel, black trace) to and stays at the bottom left stable point (basal state), when the original three stable points restores after action of $\mathrm{Ca}^{2+}$ pulses (Figure 5A, bottom right panel).

Then, we studied reversal of the established LTP by application of $\mathrm{Ca}^{2+}$ pulses. At the LTP level, the system was stable at the top left stable point (Figure 5B, left panel). During action of high $\mathrm{Ca}^{2+}$ pulses, high $\mathrm{Ca}^{2+}$ elevations deform the nullclines to create one stable state (Figure 5B, middle panel, light blue and light red 


\section{Resting $\mathrm{Ca}^{2+}$ \\ During $\mathrm{Ca}^{2+}$ influx}

A

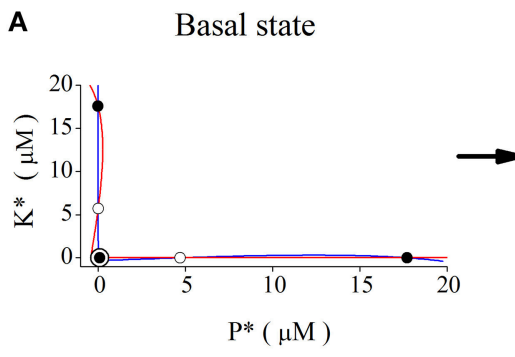

B

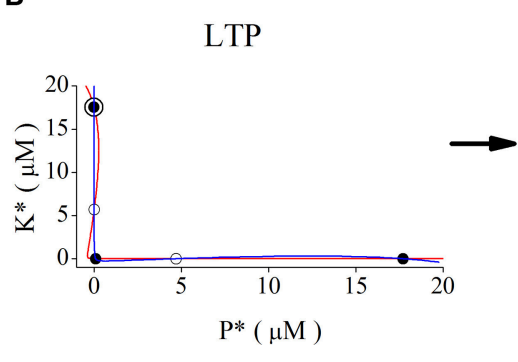

Depotentiation induction

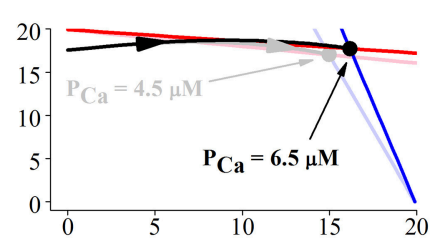

Returned to resting $\mathrm{Ca}^{2+}$
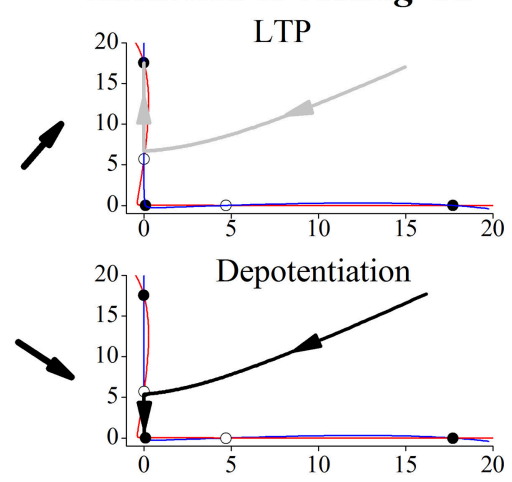

Depotentiation induction
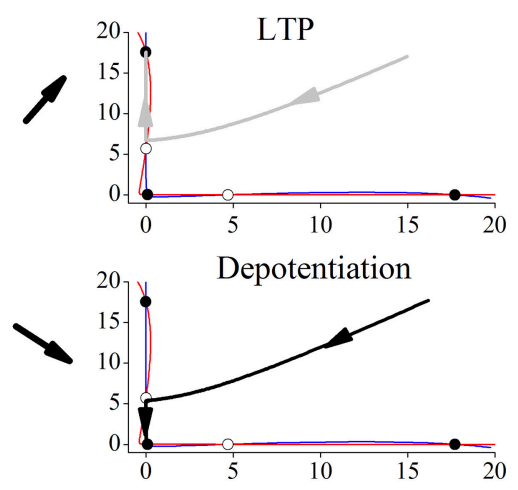

FIGURE 5 | Phase space analysis of the system's dynamics when initial state is at basal state (A) and at LTP (B), respectively. Left panel, red and blue curves indicate nullclines for kinase and phosphatase, respectively. Nullclines at basal $\mathrm{Ca}^{2+}$ concentration $(0.1 \mu \mathrm{M})$ create five steady-states. Open and filled circles denote unstable and stable steady-states, the filled circle with black open circle indicates the basal state (A) and LTP (B), $K^{*}$ and $P^{*}$ denote concentration of active kinase and phosphatase, respectively. Middle panels, nullclines during $\mathrm{Ca}^{2+}$ influx. $\mathrm{Ca}^{2+}$ elevation deforms the nullclines to create one stable state. Red and blue lines indicate nullclines for $K^{*}$ and $P^{*}$ and black dot denotes the new stable state under $6.5 \mu \mathrm{M} \mathrm{Ca}^{2+}$ elevation. Light red and light blue lines indicate nullclines for $K^{*}$ and $P^{*}$ and gray dot denotes the new stable state under $4.5 \mu \mathrm{M} \mathrm{Ca}^{2+}$ elevation. The system becomes unstable and moves to the new stable states (gray trace for $4.5 \mu \mathrm{M}$ and black trace for $6.5 \mu \mathrm{M}$ ) from basal state (A) and LTP (B), respectively. Right panels, nullclines after $\mathrm{Ca}^{2+}$ removal. Nullclines form three stable states again in the basal $\mathrm{Ca}^{2+}$ level. The state moves to the closest stable state, LTP (gray trace, top), or basal state (black trace, bottom), respectively.

lines, and gray dot), and the system moves to this new stable state (Figure 5B, middle panel, gray trace). After action of $\mathrm{Ca}^{2+}$ pulses, nullclines restore the three stable states and the system returns to the top left stable point (LTP) (Figure 5B, top right panel). During action of significant $\mathrm{Ca}^{2+}$ pulses, large $\mathrm{Ca}^{2+}$ elevations deform nullclines to create the unique stable state (Figure 5B, middle panel, blue and red lines, and black dot), and the system moves to this new stable state (Figure 5B, middle panel, black trace). After action of $\mathrm{Ca}^{2+}$ pulses, nullclines restore the three stable states, but the system moves to and stays at the bottom left stable point (basal state) (Figure 5B, bottom right panel).

Attractive basins of each stable state were depicted in the $K^{*}-P^{*}$ plane when calcium concentration was set at the basal level (Figure 6). We have known that nullclines form three stable states (filled circles at top left, bottom left, and bottom right of left panels in Figures 5A,B) at basal $\mathrm{Ca}^{2+}$ level. The $K^{*}-P^{*}$ plane is then divided into three regions; points located within the red region will evolve to the stable point at top left (named LTP basin), those in the green region to the stable point at bottom left (named basal state basin), and those in the blue region to the one point at bottom right (named LTD basin), respectively.

Then we turned to observe the distribution of the stable points created by $\mathrm{Ca}^{2+}$ elevations in the $K^{*}-P^{*}$ plane. As the amplitude of $\mathrm{Ca}^{2+}$ elevation increases from 3 to $10 \mu \mathrm{M}$ gradually, the stable points induced by $\mathrm{Ca}^{2+}$ elevation moves from bottom left to top right, from the LTP basin to the basal state basin. The stable point induced by $4.5 \mu \mathrm{M} \mathrm{Ca}^{2+}$ elevation is in the LTP basin (white dot in left), while the stable point induced by $6.5 \mu \mathrm{M}$ $\mathrm{Ca}^{2+}$ elevation is in the basal state basin (white dot in right, graph inserted in Figure 6). After the action of $\mathrm{Ca}^{2+}$ elevation, nullclines restore the three stable states, and the system will evolve from its new initial position (the position determined by $\mathrm{Ca}^{2+}$ elevation) to one of the three stable states, according to the location of this initial position in the $K^{*}-P^{*}$ plane. The new initial position determined by high $\mathrm{Ca}^{2+}$ elevation is in the LTP basin and the system evolves to LTP. The new initial position determined by significant $\mathrm{Ca}^{2+}$ elevation is in 


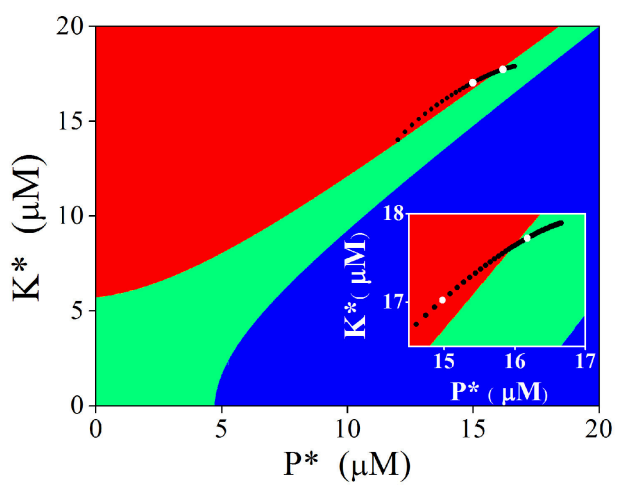

FIGURE 6 | Attractive basins with basal level $\mathrm{Ca}^{2+}$ and evolvement of the stable state induced by adjusting $\mathrm{Ca}^{2+}$ elevation. Red region indicates the attractive basin of LTP, Green for basal state, and blue for LTD. Black dots indicate evolvement of the stable state. Two white dots represent the stable states induced by $4.5 \mu \mathrm{M}$ (bottom left) and $6.5 \mu \mathrm{M}$ (top right),

respectively (also shown in the insertion).

the basal state basin, therefore, the system evolves to the basal state and can manifest in this way the depotentiation from a LTP state. Just as in the original CaMKII/PP2A network model, $\mathrm{Ca}^{2+}$ elevation is still the primary controller, which controls the synaptic strength through activation of kinase and phosphatase. In experimentations synaptic strength is not directly manipulated by $\mathrm{Ca}^{2+}$ elevation but by presynaptic simulation, including TBS. The above analysis provides a link between experiments and the generic dynamics revealed by the tristable system (Pi and Lisman, 2008).

\section{DISCUSSION}

Although there has been substantial investigation on how a tristable system composed of coupled kinase and phosphatase switches could account for stable transition of synaptic strength from basal state to both LTP and LTD, depotentiation from LTP to basal state has not been theoretically studied. Based on a series of experimental observation, the present work introduced two $\mathrm{Ca}^{2+}$ resources with different temporal dynamics, mediated by influx through NMDARs and release from internal calcium store, respectively, in the generic tristable system and further show that significant elevation of cytoplasmic calcium concentration may dynamically switch activation of both kinase and phosphatase to their basal states, thereby depotentiate the synaptic strength. The generation of this significant elevation of cytoplasmic calcium concentration depends on the length of stimulation, a prolonged stimulation activates both NMDARs and internal $\mathrm{Ca}^{2+}$ stores and then produces a significant $\mathrm{Ca}^{2+}$ elevation, while a brief stimulation only activates NMDARs and induces a high $\mathrm{Ca}^{2+}$ elevation. When initial condition of synaptic strength is at basal state, a prolonged TBS dynamically firstly induces a high $\mathrm{Ca}^{2+}$ elevation which might result in LTP, and then induces a large $\mathrm{Ca}^{2+}$ elevation and depotentiates the possible LTP.

Involvement of internal calcium store is required for induction of depotentiation. Both experiments and simulations have shown that experience exposure or stimulation protocol elevating cytoplasmic calcium concentration by activating cellular membrane calcium channels can switch the synaptic strength from basal level to LTP. We have shown in this paper that repeated experience exposure or prolonged stimulation could further activate postsynaptic group I mGluRs, produce more $I P_{3}$ to activate $\mathrm{IP}_{3} \mathrm{Rs}$ on endoplasmic reticulum, finally lead to calcium release from internal calcium store. Relatively slow dynamics of this process requires repeated experience or prolonged stimulation. Recent experiment results show that in synapses containing endoplasmic reticulum, presynaptic stimulation induced two successive elevation peaks of calcium concentration: The first one is mediated by NMDARs and the second by $\mathrm{IP}_{3}$ Rs activation, respectively(Holbro et al., 2009; Sheridan et al., 2014). These experiments support the simulation results of the present paper, not only by showing the necessity of a significant calcium elevation, but also by an equivalent time course for induction of depotentiation. The results in our modeling also suggest that depotentiation should have a threshold-like effect with calcium concentration. The prediction of this threshold and its changes with changes of internal calcium store needs to be demonstrated in further experiments.

In an interesting experiment, mutant mice lacking $\mathrm{IP}_{3} \mathrm{Rs}$ display a significantly greater magnitude of LTP induced by tetanus stimulation (Fujii et al., 2000). A similar mutant strain lacking ryanodine receptor type 3 (RYR3), another type of calcium channel on internal calcium store, exhibits in hippocampus CA1 pyramidal neurons facilitated LTP as well (Futatsugi et al., 1999). These results are controversial to some expectations for release of calcium from internal store facilitates LTP. Our simulation work could provide an explanation for these experiments, by indicating that lack of $\mathrm{IP}_{3} \mathrm{Rs}$ or RYR3 may impair calcium release, and thus prevent depotentioation, finally facilitate LTP.

Compared with the calcium elevation inducing LTP, more significant and prolonged elevation of cytoplasmic calcium concentration is required for depotentiation induction. It has been proposed that moderate elevation in $\left[\mathrm{Ca}^{2+}\right]_{c y t}$ that is produced during LTD induction may preferentially activate calcinerin, the $\mathrm{Ca}^{2+} /$ calmodulin (CaM)-dependent protein phosphatase. Calcineurin then can induce inhibition of protein 1 (I-1), the endogenous inhibitor of serine/threonine protein phosphatase-1 (PP1). This allows the activation of PP1 (Oliver and Shenolikar, 1998). PP1 dephosphorylates CaMKII and other proteins (Shields et al., 1985), including glutamate receptors, to promote LTD. However, LTP-inducing stimuli are associated with higher increase in cytoplasmic calcium concentration. This can activate CaMKII, which plays a key role in LTP induction through phosphorylation of the GluR1 subunit of AMPARs (Cormier et al., 2001). In addition, high elevation of cytoplasmic calcium concentration can activate PKA, PKA can phosphorylate and activate I-1, which then can inhibit PP1 (Barria et al., 1997). Thus, LTP is induced and LTD is prevented.

PKA, which negatively regulates $\mathrm{PP} 1$, is modulated by the activation of $\mathrm{Ca}^{2+} / \mathrm{CaM}$-dependent adenylyl cyclases (AC) (Piascik et al., 1980; Potter et al., 1980; Ahlijanian and Cooper, 
1988). And the activity of AC, which shows a bell-shaped activity curve relative to increasing calcium concentrations (Piascik et al., 1980; Potter et al., 1980; Ahlijanian and Cooper, 1988), may be decreased by large rise in cytoplasmic calcium concentration induced by prolonged TBS. The resulting low PKA activity is accompanied by a disinhibition of PP1, which inactivates CaMKII, leading occurrence of depotentiation (Lisman, 1989).

When certain memory becomes obsolete, effective extinction of the previously established memory is essential for animals to adapt to the changing environment. An exposure-based therapy for human traumatic fear memories has long been established (McNally, 2007). Fear memories present as defensive reactions to the neutral cue for a period of up to many months after learning. However, when conditioned subjects repeatedly encounter a neutral cue without a reinforcing unpleasant event, a large rise in cytoplasmic calcium concentration decreases kinase activity and subsequently induces depotentiation by AMPA receptors endocytosis (Clem and Huganir, 2010). The present simulation results may account for the mechanism of these behavioral investigations.

It has been studied and clinically applied that agonist of NMDA receptors, D-cycloserine, could facilitate fear extinction when given systematically or locally into the amygdale (Davis, 2010). Targeting mGluRs and calcium pump may provide other mechanistic insights into potential treatments for fear extinction. Moreover, significant elevation of cytoplasmic calcium concentration resulting in depotentiation is achieved by

\section{REFERENCES}

Abraham, W. C., and Huggett, A. (1997). Induction and reversal of long-term potentiation by repeated high-frequency stimulation in rat hippocampal slices. Hippocampus 7, 137-145. doi: 10.1002/(SICI)1098-1063(1997)7:2<137::AID$\mathrm{HIPO} 3>3.0 . \mathrm{CO} ; 2-\mathrm{K}$

Abraham, W. C., Logan, B., Greenwood, J. M., and Dragunow, M. (2002). Induction and experience-dependent consolidation of stable long-term potentiation lasting months in the hippocampus. J. Neurosci. 22, 9626-9634.

Ahlijanian, M. K., and Cooper, D. M. (1988). Distinct interactions between $\mathrm{Ca}^{2+} /$ calmodulin and neurotransmitter stimulation of adenylate cyclase in striatum and hippocampus. Cell. Mol. Neurobiol. 8, 459-469. doi: 10.1007/BF00711229

Barr, D. S., Lambert, N. A., Hoyt, K. L., Moore, S. D., and Wilson, W. A. (1995). Induction and reversal of long-term potentiation by low- and high-intensity theta pattern stimulation. J. Neurosci. 15, 5402-5410.

Barria, A., Muller, D., Derkach, V., Griffith, L. C., and Soderling, T. R. (1997). Regulatory phosphorylation of AMPA-type glutamate receptors by CaM-KII during long-term potentiation. Science 276, 2042-2045. doi: $10.1126 /$ science.276.5321.2042

Bikbaev, A., Neyman, S., Ngomba, R. T., Conn, P. J., Nicoletti, F., and Manahan-Vaughan, D. (2008). MGluR5 mediates the interaction between late-LTP, network activity, and learning. PLoS ONE 3:e2155. doi: 10.1371/journal.pone.0002155

Christie, B. R., Stellwagen, D., and Abraham, W. C. (1995). Reduction of the threshold for long-term potentiation by prior theta-frequency synaptic activity. Hippocampus 5, 52-59. doi: 10.1002/hipo.450050107

Clem, R. L., and Huganir, R. L. (2010). Calcium-permeable AMPA receptor dynamics mediate fear memory erasure. Science 330, 1108-1112. doi: 10.1126/science. 1195298

Collingridge, G. L., Peineau, S., Howland, J. G., and Wang, Y. T. (2010). Long-term depression in the CNS. Nat. Rev. Neurosci. 11, 459-473. doi: 10.1038/nrn2867 enzyme reactions, hence modulations on the activities of enzymes become to the second kind of approaches. It is observed that ACs is required for spatial memory extinction (Zhang et al., 2011) and mice over-expressing type $1 \mathrm{ACs}$ show enhancing spatial memory extinction (Zhang and Wang, 2013). Overall, the present findings uncover a potentially important time- and state-dependent mechanism of NMDAR- and mGluR-dependent depotentiation. Furthermore, the engagement of such depotentiation may be involved in the activity-dependent erasure of recently stored information in the hippocampus (Zhang et al., 2011; Wang and Zhang, 2012; Zhang and Wang, 2013), showing strong parallels with similar mechanisms engaged in the amygdala (Kim et al., 2007).

\section{AUTHOR CONTRIBUTIONS}

MC prepared the methods of feature construction and conducted the experiments. WR and MC prepared the manuscript. WR and XW supervised all aspects of the work. All authors discussed the results and commented on the manuscript.

\section{FUNDING}

This work was supported by the Fundamental Research Funds for the Central Universities of China (No. GK201503027 and GK201601001) and the National Natural Science Foundation of China (No. 11375109).
Cormier, R. J., Greenwood, A. C., and Connor, J. A. (2001). Bidirectional synaptic plasticity correlated with the magnitude of dendritic calcium transients above a threshold. J. Neurophysiol. 85, 399-406.

Davis, M. (2010). Facilitation of fear extinction and psychotherapy by Dcycloserine. Z. Psychol. 218, 149-150. doi: 10.1027/0044-3409/a000023

De Young, G. W., and Keizer, J. (1992). A single-pool inositol 1,4,5trisphosphate-receptor-based model for agonist-stimulated oscillations in $\mathrm{Ca}^{2+}$ concentration. Proc. Natl. Acad. Sci. U.S.A. 89, 9895-9899. doi: 10.1073/pnas.89.20.9895

Díaz-Mataix, L., Debiec, J., LeDoux, J. E., and Doyère, V. (2011). sensory-specific associations stored in the lateral amygdala allow for selective alteration of fear memories. J. Neurosci. 31, 9538-9543. doi: 10.1523/JNEUROSCI.5808-10.2011

Fujii, S., Matsumoto, M., Igarashi, K., Kato, H., and Mikoshiba, K. (2000). Synaptic plasticity in hippocampal CA1 neurons of mice lacking type 1 inositol-1,4,5trisphosphate receptors. Learn. Mem. 7, 312-320. doi: 10.1101/lm.34100

Futatsugi, A., Kato, K., Ogura, H., Li, S. T., Nagata, E., Kuwajima, G., et al. (1999). Facilitation of NMDAR-independent LTP and spatial learning in mutant mice lacking ryanodine receptor type 3. Neuron 24, 701-713. doi: 10.1016/S08966273(00)81123-X

Gruart, A., Muñoz, M. D., and Delgado-García, J. M. (2006). Involvement of the CA3-CA1 synapse in the acquisition of associative learning in behaving mice. J. Neurosci. 26, 1077-1087. doi: 10.1523/JNEUROSCI.2834-05.2006

Holbro, N., Grunditz, A., and Oertner, T. G. (2009). Differential distribution of endoplasmic reticulum controls metabotropic signaling and plasticity at hippocampal synapses. Proc. Natl. Acad. Sci. U.S.A. 106, 15055-15060. doi: 10.1073/pnas.0905110106

Kandel, E. R., and Spencer, W. A. (1961). Electrophysiology of hippocampal neurons. II. After-potentials and repetitive firing. J. Neurophysiol. 24, 243-259.

Kang-Park, M. H., Sarda, M. A., Jones, K. H., Moore, S. D., Shenolikar, S., Clark, S., et al. (2003). Protein phosphatases mediate depotentiation induced by high-intensity theta-burst stimulation. J. Neurophysiol. 89, 684-690. doi: 10.1152/jn.01041.2001 
Kim, J., Lee, S., Park, K., Hong, I., Song, B., Son, G., et al. (2007). Amygdala depotentiation and fear extinction. Proc. Natl. Acad. Sci. U.S.A. 104, 20955-20960. doi: 10.1073/pnas.0710548105

Kusters, J. M., Dernison, M. M., van Meerwijk, W. P., Ypey, D. L., Theuvenet, A. P., and Gielen, C. C. (2005). Stabilizing role of calcium store-dependent plasma membrane calcium channels in action-potential firing and intracellular calcium oscillations. Biophys. J. 89, 3741-3756. doi: 10.1529/biophysj.105.062984

Li, S., Cullen, W. K., Anwyl, R., and Rowan, M. J. (2003). Dopamine-dependent facilitation of LTP induction in hippocampal CA1 by exposure to spatial novelty. Nat. Neurosci. 6, 526-531. doi: 10.1038/nn1049

Lisman, J. (1989). A mechanism for the Hebb and the anti-Hebb processes underlying learning and memory. Proc. Natl. Acad. Sci. U.S.A. 86, 9574-9578. doi: $10.1073 /$ pnas.86.23.9574

Madroñal, N., Delgado-García, J. M., and Gruart, A. (2007). Differential effects of long-term Potentiation evoked at the CA3-CA1 synapse before, during, and after the acquisition of classical eyeblink conditioning in behaving mice. J. Neurosci. 27, 12139-12146. doi: 10.1523/JNEUROSCI.3397-07.2007

Manahan-Vaughan, D., and Braunewell, K. H. (1999). Novelty acquisition is associated with induction of hippocampal long-term depression. Proc. Natl. Acad. Sci. U.S.A. 96, 8739-8744. doi: 10.1073/pnas.96.15.8739

McKernan, M. G., and Shinnick-Gallagher, P. (1997). Fear conditioning induces a lasting potentiation of synaptic currents in vitro. Nature 390, 607-611. doi: $10.1038 / 37605$

McNally, R. J. (2007). Mechanisms of exposure therapy: how neuroscience can improve psychological treatments for anxiety disorders. Clin. Psychol. Rev. 27, 750-759. doi: 10.1016/j.cpr.2007.01.003

Oliver, C. J., and Shenolikar, S. (1998). Physiologic importance of protein phosphatase inhibitors. Front. Biosci. 3, D961-D972.

Pi, H. J., and Lisman, J. E. (2008). Coupled phosphatase and kinase switches produce the tristability required for long-term potentiation and long-term depression. J. Neurosci. 28, 13132-13138. doi: 10.1523/JNEUROSCI.234808.2008

Piascik, M. T., Wisler, P. L., Johnson, C. L., and Potter, J. D. (1980). Ca ${ }^{2+}$ dependent regulation of guinea pig brain adenylate cyclase. J. Biol. Chem. 255, 4176-4181.

Potter, J. D., Piascik, M. T., Wisler, P. L., Robertson, S. P., and Johnson, C. L. (1980). Calcium dependent regulation of brain and cardiac muscle adenylate cyclase. Ann. N. Y. Acad. Sci. 356, 220-231. doi: 10.1111/j.1749-6632.1980. tb29613.x

Qi, Y. J., Hu, N. W., and Rowan, M. J. (2013). Switching off LTP: mGlu and NMDA receptor-dependent novelty exploration-induced depotentiation in the rat hippocampus. Cereb. Cortex. 23, 932-939. doi: 10.1093/cercor/ bhs086

Ranck, J. B. Jr. (1973). Studies on single neurons in dorsal hippocampal formation and septum in unrestrained rats. I. Behavioral correlates and firing repertoires. Exp. Neurol. 41, 461-531.
Rogan, M. T., Stäubli, U. V., and LeDoux, J. E. (1997). Fear conditioning induces associative long-term potentiation in the amygdala. Nature 390, 604-607. doi: $10.1038 / 37601$

Rumpel, S., LeDoux, J., Zador, A., and Malinow, R. (2005). Postsynaptic receptor trafficking underlying a form of associative learning. Science 308, 83-88. doi: 10.1126/science. 1103944

Sheridan, G. K., Moeendarbary, E., Pickering, M., O'Connor, J. J., and Murphy, K. J. (2014). Theta-burst stimulation of hippocampal slices induces networklevel calcium oscillations and activates analogous gene transcription to spatial learning. PLoS ONE 9:e100546. doi: 10.1371/journal.pone.0100546

Shields, S. M., Ingebritsen, T. S., and Kelly, P. T. (1985). Identification of protein phosphatase 1 in synaptic junctions: dephosphorylation of endogenous calmodulin-dependent kinase II and synapse-enriched phosphoproteins. J. Neurosci. 5, 3414-3422.

Shouval, H. Z., Bear, M. F., and Cooper, L. N. (2002). A unified model of NMDA receptor-dependent bidirectional synaptic plasticity. Proc. Natl. Acad. Sci. U.S.A. 99, 10831-10836. doi: 10.1073/pnas.152343099

Straube, T., Korz, V., and Frey, J. U. (2003). Bidirectional modulation of long-term potentiation by novelty-exploration in rat dentate gyrus. Neurosci. Lett. 344, 5-8. doi: 10.1016/S0304-3940(03)00349-5

Wang, H. B., and Zhang, M. (2012). The role of $\mathrm{Ca}^{2+}$-stimulated adenylyl cyclases in bidirectional synaptic plasticity and brain function. Nat. Neurosci. 23, 67-78. doi: 10.1515/revneuro-2011-0063

Whitlock, J. R., Heynen, A. J., Shuler, M. G., and Bear, M. F. (2006). Learning induces long-term potentiation in the hippocampus. Science 313, 1093-1097. doi: $10.1126 /$ science.1128134

Xu, L., Anwyl, R., and Rowan, M. J. (1998). Spatial exploration induces a persistent reversal of long-term potentiation in rat hippocampus. Nature 394, 891-894. doi: $10.1038 / 29783$

Zhang, M., Storm, D. R., and Wang, H. B. (2011). Bidirectional synaptic plasticity and spatial memory flexibility require $\mathrm{Ca}^{2+}$-stimulated adenylyl cyclases. J. Neurosci. 31, 10174-10183. doi: 10.1523/JNEUROSCI.0009-11.2011

Zhang, M., and Wang, H. (2013). Mice overexpressing type 1 adenylyl cyclase show enhanced spatial memory flexibility in the absence of intact synaptic long-term depression. Learn. Mem. 20, 352-357. doi: 10.1101/lm.030114.112

Conflict of Interest Statement: The authors declare that the research was conducted in the absence of any commercial or financial relationships that could be construed as a potential conflict of interest.

Copyright (C) 2016 Chen, Ren and Wang. This is an open-access article distributed under the terms of the Creative Commons Attribution License (CC BY). The use, distribution or reproduction in other forums is permitted, provided the original author(s) or licensor are credited and that the original publication in this journal is cited, in accordance with accepted academic practice. No use, distribution or reproduction is permitted which does not comply with these terms. 